\title{
Comparison of Left and Right Fingertip PPG Signals Using Signal Power Estimates and Poincare Indexes
}

\author{
Surhan Bozkurt, Gokhan Ertas \\ Department of Biomedical Engineering, Yeditepe University, Istanbul, Turkey \\ Email address: \\ surhan.bozkurt@yeditepe.edu.tr (S. Bozkurt),gokhan.ertas@yeditepe.edu.tr (G. Ertas)
}

To cite this article:

Surhan Bozkurt, Gokhan Ertas. Comparison of Left and Right Fingertip PPG Signals Using Signal Power Estimates and Poincare Indexes. American Journal of Biomedical and Life Sciences. Special Issue: Numerical and Experimental Research in Cardiovascular Sciences. Vol. 3, No. 2-2, 2015, pp. 6-10. doi: 10.11648/j.ajbls.s.2015030202.12

\begin{abstract}
Photoplethysmography (PPG) is a non-invasive method to measure the relative blood volume change in blood vessels used in a wide range of medical applications. PPG signals can be recorded from different body regions such as fingertips, forehead and earlobes. In this study, information content of PPG signals of the left and the right index fingertips are processed and analyzed. PPG recordings are performed from ten healthy volunteers. Prior to recordings, all volunteers take rest for five minutes. Using a dedicated measurement system, signals are recorded from the left and the right fingertips of each volunteer simultaneously for 60 seconds with a sampling frequency of $50 \mathrm{~Hz}$, digitized with 10-bit resolution and stored in a personal computer. Signal average power and Poincare indexes of the signals are estimated and statistically analyzed. There are no systematic differences between the signal power estimated from the left and right fingertip signals $(P>0.5)$. The signal power estimates for the left and the right fingertips show moderate correlations of $0.78,0.80$ and 0.68 for $0-4 \mathrm{~Hz}, 0-2 \mathrm{~Hz}$ and $2-4 \mathrm{~Hz}$ frequency bands, respectively. When Poincare indexes $S D 1$ and $S D 1 / S D 2$ are considered, there are no systematic differences between the left and right fingertip signals $(P>0.5)$ however a systematic difference exists between the $S D 2$ estimates $(P=0.15)$. $S D 1$ and $S D 1 / S D 2$ estimates for the left and the right fingertips show high positive correlations of 1.00 and 0.99 , respectively. However, a correlation of -0.30 exits for the left and the right fingertips when $S D 2$ estimate is considered.
\end{abstract}

Keywords: Photoplethysmography, Fingertip, Signal Power, Poincaré

\section{Introduction}

Photoplethysmography (PPG) is a non-invasive method based on light absorption to measure relative blood volume change in blood vessels and has been used in a wide range of medical applications to measure oxygen saturation, cardiac output and blood pressure in arterial blood vessel [1] and also to estimate the arterial stiffness from forearm $[2,3]$. In this technique, an infrared light source provides infrared light radiation that penetrates under the skin surface at different human body locations such as fingertip, forehead and earlobe [4]. The intensity of transmitted or backscattered light is measured by optically sensitive electronic devices such as phototransistors.

There are several studies on processing of PPG signals to get some physiological information. Lu et al. analyzed heart rate variability and PPG variability by computing several time and frequency domain parameters of both signals. The results show that the parameters of PPG variability are highly correlated with the parameters of heart rate variability [5]. Yoon et al. studied PPG signals to predict blood pressure and used systolic upstroke time and diastolic time parameters extracted from the PPG signals of five volunteers [6]. The time differences in the systolic times detected on the PPG signals are studied to make correlations with physiological stress of human. It has been reported that the time difference can be used as an index in predicting stress level [7]. PPG signals from five healthy volunteers and five cardiovascular patients are studied to determine heart rate variability and the results show that PPG signals are beneficial in analysis of cardiovascular disorders supplementing the existing methods [8]. Ye et al. proposed a method for non-invasive but indirect blood pressure measurements based on pulse transit time of ECG and PPG signals from volunteers. When blood pressure estimated indirectly by the proposed algorithm compared with the blood pressure measured by a conventional sphygmomanometer, the results indicate a mean error of \pm 3.24 $\mathrm{mmHg}$ and a standard deviation of $2.53 \mathrm{mmHg}$ [9]. Gescge et al. studied pulse transit time and pulse wave velocity of PPG 
signals and systolic blood pressure recorded from 63 volunteers. Results show relevant differences [10]. Bharati et al. analyzed the PPG signals from twenty-one diabetes, arthritis and healthy volunteers. The results shows that PPG signal waveform has very small dichroitic notch and slope is less, whereas arthritis patients PPG waveform has very sharp slope no dichroitic notch. [11]. Pilt et al. used Adaptive Sum Comb Filter for the AC component of the PPG signal. The results show that adjusted Comb filter has about $7 \mathrm{~dB}$ better noise attenuation than non-adjusted Comb filter by using same number of recurrences for filter output calculation [12]. Gil et al. analyzed heart rate variability (HRV) during decreases in the amplitude fluctuations in PPG signals for obstructive sleep apnea syndrome. Results show that amplitude fluctuations in PPG signals are not associated with either respiratory events or $\mathrm{SpO}_{2}$ decrement. Heart rate variability shows slight alternations and the signal power is shifted to higher frequencies. Decreases in the amplitude fluctuations of Photoplethysmography (DAP) associated with apnea lead to stronger heart rate [14].

PPG signals may suffer from motion during signal acquisitions that leads to undesirable decreases in measurement accuracy. To eliminate motion, Yao et al. proposed a generalized method based on Beer-Lambert Law that requires acquisition of Photoplethysmography data acquired at three excitation wavelength [13]. Kim et al. proposed a method to obtain accurate PPG signals from finger and forehead in the presence of constrained patient motion. Motion is detected by using an accelerometer and distorted PPG signal is corrected using the motion information and adaptive filtering. Corrected PPG signal is reported to be with similar accuracy obtained by motionless finger PPG signal [15]. Sarkar et al. proposed a method in detecting heart rate and respiration rate from motion induced PPG signals using wavelets. The results show that there is a difference in the pulse shape changes as a function of disease [16]. To remove motion artifacts on dynamic PPG signals, Chan et al. used and compared variable step size and fixed step size filters based on Least Mean Square. The variable step size Least Mean Square filter provides better performance than the Least Mean Square filter with fixed step size [17].

In this study, we aim to analyze PPG signals from right and left fingertips to make relations between the signals in terms of signal power and Poincare indexes.

\section{Materials and Methods}

In this study, PPG signals are recorded from the left and the right fingertips simultaneously by using KL-7001 Biomedical Measurement System (K\&H MFG Co. LTD). This measurement system is equipped with an infrared sensor avoiding the interference of visible environmental light, a second order high pass filter eliminating the drift voltage due to the tremble of finger or DC biasing voltage of infrared sensor and an amplifier circuitry to amplify the filtered signal avoiding signal distortions. A fourth order low pass filter eliminates the interference of power source and avoids the high frequency noise. A differentiator accentuates the minute changes in the signal.

Ten healthy volunteers (five male and five female; mean age: 23.4 years) were enrolled in the study. All volunteers took rest for 5 minutes before signal recordings. Recordings were performed for a period of 60 seconds with a sampling frequency of $50 \mathrm{~Hz}$. The signals recorded were next digitized with 10-bit resolution and stored in a personal computer for further analysis. During analysis signal power estimates and Poincare indexes were computed using Matlab 2013 (The Mathwork Inc., Natick, MA).

\subsection{Signal Power Estimates}

A non-parametric estimation of signal power is provided by power spectral density of finite-length signals by Periodogram method based on fast Fourier transformation [18]. Let $x(n)$ be the time series data of a PPG signal. Let $N$ and $f$ be the total number of samples and the signal frequency, respectively. Power spectral density, $P$ is estimated using

$$
\hat{P}(f)=\frac{1}{N}\left|\sum_{n=0}^{N-1} x(n) e^{-j 2 \pi f n}\right|^{2}
$$

In this study, using the power spectral density estimated for the left fingertip and the right fingertip PPG signals from each volunteer, the signal power for frequency bands of $0-4 \mathrm{~Hz}, 0-2$ $\mathrm{Hz}$ and $2-4 \mathrm{~Hz}$ are determined.

\subsection{Poincare Indexes}

Poincare analysis are originally defined for the RR interval values determined for ECG signals in order to study the behavior of the RR interval variability [19]. Usually, a 2D Poincare plot is provided to depict the correlation between consecutive intervals in a graphical representation. Visual examination of the Poincare plot shapes provides valuable qualitative information in discriminating several heart diseases or conditions [19]. In addition to this, quantitative information can be gathered by performing mathematical analysis giving there Poincare indexes, namely $S D 1, S D 2$ and $S D 1 / S D 2$. In this study, we apply Poincare analysis on the PPG signals recorded.

Let $X$ be the time series data holding the values of L-number of peak intervals detected for a PPG signal. Now, let $S D S D$ and $S D X$ are the standard deviation of the successive difference of time series data $\left(X_{i+1}-X_{i}\right)(i=1,2, \ldots ; L-1)$ and the standard deviation of $X$ respectively. $S D 1$ and $S D 2$ are given by

$$
\begin{gathered}
S D 1=\frac{1}{\sqrt{2}} S D S D \\
S D 2=\sqrt{2 S D X^{2}-S D 1^{2}}
\end{gathered}
$$

$S D 1$ is considered to describe the short-term variability and SD2 the long-term variability in PPG peak signal variability analysis. Overall SD1 and SD2 change tremendously with increasing number of peak intervals. The third index $S D 1 / S D 2$ is derived from the ratio of $S D 1$ to $S D 2$. 


\subsection{Statistics}

To compare the information content of the PPG signals recorded from left and tight fingertips, the descriptors of the spectral and Poincare analyses extracted were analyzed. Systematic differences between the descriptors estimated from the left and the right fingertips were tested using independent samples t-test with either a pooled or a separate variance as determined by the Levene's test for equality of variances. A $P$ value $<0.5$ was considered to be statistically significant. The Pearson correlation coefficient $r$ was calculated to determine the correlation between the descriptors estimated from the left and the right fingertips. All statistical analyses were performed using SPSS 17 (SPSS Inc., USA).

\section{Results}

The PPG signals recorded from a male volunteer is considered for demonstrative purposes (see Figure 1). Figures $2 \mathrm{a}$ and $2 \mathrm{~b}$ show the signals recorded from the left and the right fingertips, respectively. Figure 3 shows the power spectral density spectrum computed for the signals. On these plots, two peaks are noticeable close to $1.2 \mathrm{~Hz}$ and $2.7 \mathrm{~Hz}$. For the left fingertip PPG signal, the signal power estimates are 4.08, 3.77 and 0.31 for $0-4 \mathrm{~Hz}, 0-2 \mathrm{~Hz}$ and $2-4 \mathrm{~Hz}$ frequency bands, respectively. Right fingertip PPG signal powers are estimated as $6.29,5.34$ and 0.95 for $0-4 \mathrm{~Hz}, 0-2 \mathrm{~Hz}$ and $2-4 \mathrm{~Hz}$ frequency bands, respectively. From the left fingertip signal, SD1, SD2 and SD1/SD2 indexes computed are 2.14, 1228.70 and 1.00, respectively. SD1, SD2 and SD1/SD2 index values are 2.16, 1228.50 and 1.10 , respectively for the right fingertip.

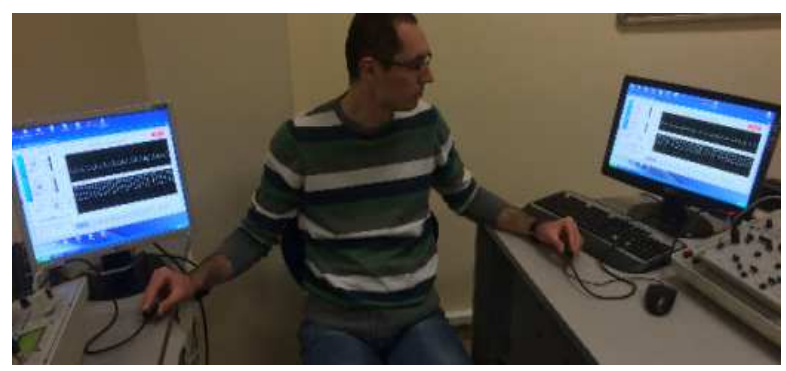

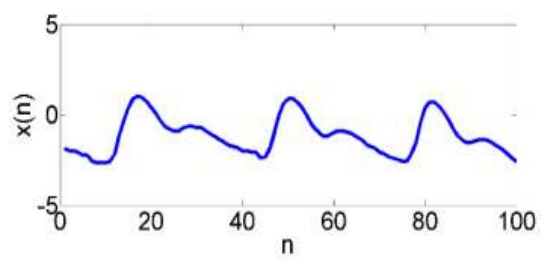

(a)

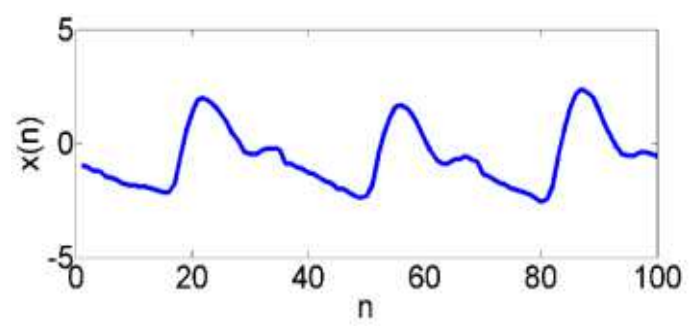

(b)

Figure 2. PPG signals of a healthy volunteer recorded from a) the left and b) the right fingertips.

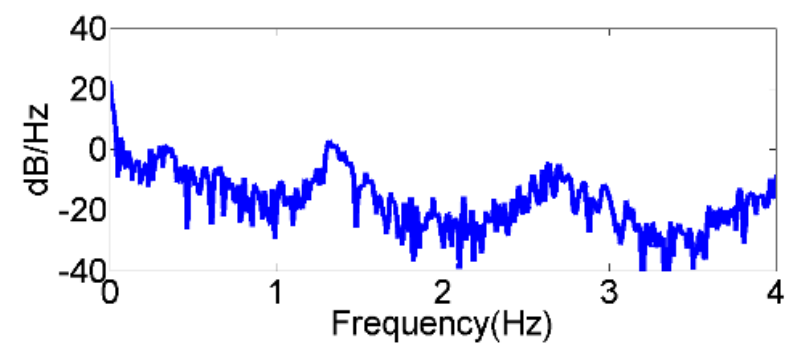

(a)

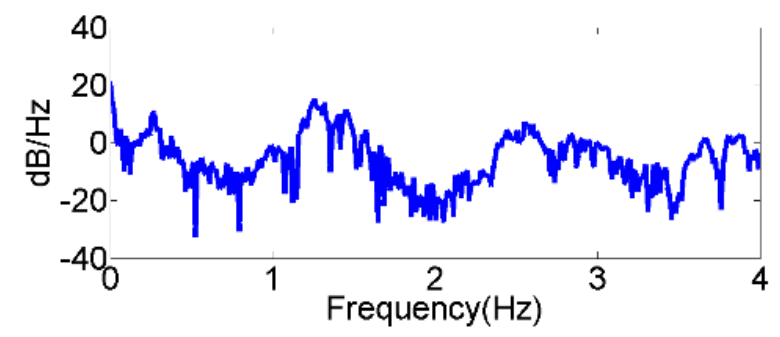

(b)

Figure 3. Power spectral density spectrum of the a) left fingertip signal and b) right fingertip signal.

Figure 1. Simulatneous $P P G$ signal recording from the left and the right index fingertips of a male volunteer.

Table 1. Average power estimates for frequency bands

\begin{tabular}{lllllll}
\hline \multirow{2}{*}{ Volunteer } & $\widehat{\boldsymbol{P}}_{\mathbf{0 - 4 H z}}$ & & $\widehat{\boldsymbol{P}}_{\mathbf{0}-\mathbf{H z}}$ & & $\widehat{\boldsymbol{P}}_{\mathbf{2}-\mathbf{H z}}$ \\
\cline { 2 - 7 } & Left Fingertip & Right Fingertip & Left Fingertip & Right Fingertip & Left Fingertip & Right Fingertip \\
\hline$\# 1$ & 4.38 & 5.59 & 4.23 & 5.23 & 0.15 & 0.36 \\
$\# 2$ & 6.71 & 5.22 & 5.72 & 4.45 & 0.99 & 0.75 \\
$\# 3$ & 2.85 & 3.58 & 2.61 & 3.35 & 0.24 & 0.23 \\
$\# 4$ & 4.09 & 6.29 & 3.77 & 5.34 & 0.31 & 0.95 \\
$\# 5$ & 5.14 & 5.77 & 4.61 & 4.96 & 0.53 & 0.81 \\
$\# 6$ & 3.45 & 4.16 & 3.39 & 4.06 & 0.06 & 0.10 \\
$\# 7$ & 2.95 & 2.31 & 2.68 & 2.28 & 0.27 & 0.03 \\
$\# 8$ & 2.96 & 3.22 & 2.88 & 2.92 & 0.08 & 0.30 \\
$\# 10$ & 2.02 & 2.46 & 1.97 & 2.32 & 0.05 & 0.14 \\
\hline
\end{tabular}


The signal power estimates for the PPG signals of all volunteers recorded from the left and the right fingertips are as presented in Table 1. For the left fingertip, signal power estimated for the frequency bands are $3.66 \pm 1.46(0-4 \mathrm{~Hz})$, $3.39 \pm 1.21(0-2 \mathrm{~Hz})$ and $0.28 \pm 0.30(2-4 \mathrm{~Hz})$. For right fingertip, signal powers estimated are $4.06 \pm 1.57(0-4 \mathrm{~Hz}), 3.69 \pm 1.28$ $(0-2 \mathrm{~Hz})$ and $0.36 \pm 0.40(2-4 \mathrm{~Hz})$. There are no systematic differences between the signal power estimated from the left and right fingertips for all frequency bands $(P>0.5)$. In addition to these, the signal power estimates for the left and the right fingertips show moderate degree of correlations: $r=0.78, \quad 0.80$ and 0.68 for $0-4 \mathrm{~Hz}, 0-2 \mathrm{~Hz}$ and $2-4 \mathrm{~Hz}$ frequency bands, respectively.

Poincare indexes computed for the PPG signals of all volunteers recorded from the left and the right fingertips are seen in Table 2. On average, SD1, SD2 and SD1/SD2 estimated for the left fingertip PPG signals are $4.84 \pm 4.70$, $1206.52 \pm 67.59$ and $0.01 \pm 0.01$, respectively. On the other hand, for the right fingertip signals, average $S D 1, S D 2$ and $S D 1 / S D 2$ are computed as $4.77 \pm 4.61,1239.67 \pm 18.91$ and $0.01 \pm 0.01$, respectively. There are no systematic differences between the Poincare indexes $S D 1$ and $S D 1 / S D 2$ estimated for the left and right fingertips $(P>0.5)$ while a systematic difference exists between $S D 2$ estimates for the left and right fingertips $(P=0.15)$. Among the Poincare indexes, $S D 1$ and $S D 1 / S D 2$ estimates for the left and the right fingertips show high positive correlations of 1.00 and 0.99 , respectively. However, a correlation of -0.30 exits for the left and the right fingertips for the $S D 1$ estimate.

Table 2. Poincaré indexes computed.

\begin{tabular}{|c|c|c|c|c|c|c|}
\hline \multirow{2}{*}{ Volunteer } & \multicolumn{2}{|l|}{ SD1 } & \multicolumn{2}{|l|}{ SD2 } & \multicolumn{2}{|l|}{$S D 1 / S D 2\left(\times 10^{-3}\right)$} \\
\hline & Left Fingertip & Right Fingertip & Left Fingertip & Right Fingertip & Left Fingertip & Right Fingertip \\
\hline \# 1 & 1.86 & 1.97 & 1232.40 & 1230.80 & 1.50 & 1.60 \\
\hline \#2 & 2.46 & 1.82 & 1244.70 & 1215.50 & 2.00 & 1.50 \\
\hline \# 3 & 1.96 & 1.81 & 1241.50 & 1260.00 & 1.60 & 1.50 \\
\hline \# 4 & 2.14 & 2.16 & 1228.70 & 1228.50 & 1.70 & 1.80 \\
\hline \# 5 & 1.27 & 1.36 & 1219.80 & 1219.80 & 1.00 & 1.10 \\
\hline \# 6 & 11.92 & 12.34 & 1241.00 & 1240.50 & 9.60 & 9.90 \\
\hline \# 7 & 1.61 & 1.65 & 1249.20 & 1249.80 & 1.30 & 1.30 \\
\hline \# 8 & 4.01 & 4.01 & 1236.20 & 1039.60 & 3.90 & 3.20 \\
\hline \# 9 & 6.80 & 7.20 & 1218.20 & 1242.90 & 5.50 & 5.90 \\
\hline \# 10 & 14.37 & 13.35 & 1285.00 & 1137.80 & 12.60 & 10.40 \\
\hline
\end{tabular}

\section{Discussion and Conclusion}

PPG signals can be recorded under the skin surface at different sites of human body such as fingertip, forehead and earlobe. In this study, PPG signals are recorded from the left and the right index fingertips simultaneously. The signals are processed and the signal power and the Poincare indexes are estimated to make comparisons.

Determination of the signal power based on Periodograms provide beneficial information but requiring proper and long-time PPG recordings. If the PPG signal does not cover a whole number of cycles within the time window, "spectral leakage effect" distortion may occur. Therefore, precautions must be taken while computing Periodograms. In some situations, manual truncation of the signal should be performed. Poincare indexes have been originally proposed to study the behavior of the RR interval variability on ECG signals in discriminating several heart diseases or conditions. However, this study shows that these indexes can be also used in the analysis of PPG signals providing valuable quantitative information inherently.

Analyses of PPG signals from ten healthy volunteers show that there are no systematic differences between the signal power estimated from the signals recorded from the left and the right index fingertips. However, signal power estimates for the left and the right fingertips show moderate correlations ( 0.80 at maximum). There are no systematic differences between Poincare indexes SD1 and SD1/SD2 estimated for the left and right fingertips while a systematic difference exists between SD2 estimates. However, SD1 and $S D 1 / S D 2$ estimates for the left and the right fingertips show very high positive correlations ( 0.99 at minimum). A negative correlation exits for the left and the right fingertips for SD1 index.

In the future, we plan to study the "features" mentioned above on the forehead and chest PPG recordings. We also plan to expand our dataset to include PPG recordings from patients suffering from cardiovascular problems and to study the imbalances of the "features" as an indicator of healthiness.

\section{References}

[1] J. G. Webster, ed. Design of Pulse Oximeters (Institute of Physics Publishing, Bristol and Philadelphia, 1997), pp. 13-39.

[2] J. Allan and A. Murray, "Age-related changes in peripheral pulse timing characteristics at the ears, finger and toe", J. Human Hypertension, vol. 16, pp. 711-717, 2005.

[3] C. Choi, K-S Soh, S. M. Lee an G. Yoon, "Propagation of light along an acupuncture meridian", Journal of the Optical Society of Korea, vol. 7, no. 4, pp.245-248, 2003.

[4] J. Spiguilis, "Optical noninvasive monitoring of skin blood pulsation." Applied Optics. Vol. 44, no. 10, pp. 1850-1857, 2005. 
[5] Lu S, Zhao H, Ju K, Shin K, Lee M, Shelley K, Chon KH, “Can photoplethysmography variability serve as an alternative approach to obtain heart rate variability information", Journal of Clinical Monitoring and Computing, 2008 Feb. 22(1):23-9

[6] Y. Yoon and G. Yoon, "Nonconstrained Blood Pressure Measurement by Photoplethysmography", Journal of the Optical Society of Korea, Vol. 10, No.2, June 2006, pp 91-95.

[7] S. Hey, A. Garbi, B. von Harren, K. Walter, N. Könning and S. Löffler, "Continuous non-invasive Pulse Transmit Time Measurement for Physiological Stress Monitoring", Proceeding of International Conference on eHealt, Telemedicine, and Social Medicine, eTELEMED 2009, Cancun, Feb. 2009, IEEE Computer Society Conference Proceedings, pp 113-116.

[8] V. S. Murthy , S. Ramamoorthy, N. Srinivasan, S. Rajagopal and M. M. Rao, "Analysis of Photoplethysmographic Signals of Cardiovascular Patients", Engineering in Medicine and Biology Society, 2001, Proceedings of the 23rd Annual International Conference of the IEEE, Vol. 3, pp 2204-2207.

[9] Soo-young Ye, Gi-Ryon Kim, Dong Keun Jung, Seong-wan Baik and Gye-rok Jeon, "Estimation of Systolic and Diastolic Pressure using the Pulse Transmit Time", Word Academy of Science, Engineering and Technology, Vol. 4, No. 7, 2010.

[10] H. Gesche, D. Grosskurth, G. Küchler and A. Patzak, "Continous Blood Pressure Measurement by Using Pulse Transmit Time: Comparison to a Cuff-based Method", European Journal of Applied Physiology, 2011.

[11] S. Bharati and G. Gidveer, "Wave form analysis of Pulse Wave detected in fingertip with PPG", International Journal of Advances in Engineering and Technology, March 2012, Vol. 3, Isssue 1, pp 92-100.

[12] K. Pilt, K. Meigas, R. Ferenets and J. Kaik, "Adjustment of Adaptive Comb Filter for PPG Signals", 31st Annual
International Conferences of the IEEE EMBS Minneapolis, Minnesota, USA, September 2-6, 2009.

[13] J. Yao and S. Warren, "A Novel Algorithm to Seperate Motion Artifacts from Photopletysmographic Signals Obtained with a Reflectance Pulse Oximeter", Proceeding of the 26th Annual International Conferences of the IEEE EMBS, San Francisco, CA, USA, September 1-5, 2004.

[14] E. Gil, M. Mendez, J. M. Vergara and S. Cerutti, "Discrimination of Sleep-Apnea-Related Decreases in the Amplitude Fluctuations of PPG Signal in Children by HRV Analysis", IEEE Transactions on Biomedical Engineering, Vol. 56, No. 4, April 2009.

[15] Sang Hyun Kim, Dong Wan Ryoo and Changseok Bae, "Adaptive Noise Cancellation Using Accelerometers for the PPG Signals from Forehead", Proceeding of the 29th Annual International Conference of the IEEE EMBS Cite Internationale, Lyon, France, August, 23-26, 2007

[16] S. Sarkar, A. K. Bhoi and G. Sativa, "Fingertip Pulse Wave (PPG Signal) Analysis and Heart Rate Detection", International Journal of Emerging Technology and Advanced Engineering, Vol. 2, pp 404-408, 2012.

[17] K. W. Chan and Y. T. Zhang, "Adaptive Reduction of Motion Artifact from Photoplethysmographic Recordings using a Variable Step-Size LMS Filter", Sensors, Proceeding of IEEE, Vol. 2, pp 1343-1346, 2002.

[18] P. S. R. Diniz, E. A. B. da Silva and S. L. Netto, "Digital Signal Processing, Sytem Analysis and Design", Second Edition, Federal University of Rio de Janerio, 2010.

[19] Che-Hao H, Ming-Ya T, Go-Shine H, Tso-Chou L, Kuen-Pao C, Shung-Tai H, Liyang-Yu S, Chi-Yuan L, "Poincaré plot indexes of heart rate variability detect dynamic autonomic modulation during general anesthesia induction", Acta Anaesthesiologica Taiwanica 50 (2012) 12-18 